\title{
Gadofosveset-enhanced MRI as simple surrogate parameter for real-time evaluation of the initial tumour vessel infarction by retargeted tissue factor tTF-NGR
}

\author{
ANNA HÖINK ${ }^{1,2}$, THORSTEN PERSIGEHL ${ }^{1,2}$, ROBERT KWIECIEN $^{3}$, MARTIN BALTHASAR $^{2}$, ROLF MESTERS $^{4}$, \\ WOLFGANG BERDEL ${ }^{4}$, WALTER HEINDEL ${ }^{1}$, CHRISTOPH BREMER ${ }^{1,5}$ and CHRISTIAN SCHWÖPPE ${ }^{5}$ \\ ${ }^{1}$ Department of Clinical Radiology, University Hospital Münster, D-48149 Münster; \\ ${ }^{2}$ Department of Diagnostic and Interventional Radiology, University Hospital Cologne, D-50937 Cologne; \\ ${ }^{3}$ Institute of Biostatistics and Clinical Research, University of Münster; ${ }^{4}$ Department of \\ Medicine A - Haematology and Oncology, University Hospital Münster, D-48149 Münster; \\ ${ }^{5}$ Department of Radiology, St. Franziskus-Hospital Münster, D-48145 Münster, Germany
}

Received March 28,2018; Accepted August 22, 2018

DOI: $10.3892 / \mathrm{ol} .2018 .9638$

\begin{abstract}
Truncated tissue factor (tTF)-NGR consists of the extracellular domain of the human TF and the binding motif NGR. tTF-NGR activates blood coagulation within the tumour vasculature following binding to CD13, and is overexpressed in the endothelial cells of tumour vessels, resulting in tumour vessel infarction and subsequent retardation/regression of tumour growth. The aim of the present study was to investigate gadofosveset-based real-time dynamic contrastenhanced magnetic resonance imaging (DCE-MRI) in evaluating the initial therapeutic effects of the anti-vascular tTF-NGR approach. DCE-MRI (3.0 T) was performed in human U87-glioblastoma tumour-bearing nude mice. During a dynamic T1w GE-sequence, a gadolinium-based blood pool contrast agent (gadofosveset) was injected via a tail vein catheter. Following the maximum contrast intensity inside the tumour being obtained, tTF-NGR was injected (controls received $\mathrm{NaCl}$ ) and the contrast behaviour of the tumour was monitored by ROI analysis. The slope difference of signal intensities between controls and the tTF-NGR group was investigated, as well as the differences between the average area under the curve (AUC) of the two groups. The association between intensity, group (control vs. tTF-NGR group) and time was analysed by fitting a linear mixed model. Following the injection of tTF-NGR, the signal intensity inside the tumours exhibited a statistically significantly stronger average slope decrease compared with the signal intensity of the tumours in
\end{abstract}

Correspondence to: Dr Anna Höink, Department of Diagnostic and Interventional Radiology, University Hospital Cologne, Building 18 a, Kerpener Straße 62, D-50937 Cologne, Germany E-mail: anna.hoeink@uk-koeln.de

Key words: gadofosveset, anti-vascular treatment, truncated tissue factor, tumour vessel infarction, real-time visualization the $\mathrm{NaCl}$ group. Furthermore, the initial average AUC values of mice treated with $\mathrm{tTF}-\mathrm{NGR}$ were $5.7 \%$ lower than the average AUC of the control animals $(\mathrm{P}<0.05)$. Gadofosveset-enhanced MRI enables the visualization of the initial tumour response to anti-vascular treatment in real-time. Considering the clinical application of tTF-NGR, this method may provide a simple alternative parameter for monitoring the tumour response to vascular disrupting agents and certain vascular targeting agents in humans.

\section{Introduction}

Over the past few years, cancer therapy has been subject to continuous improvement. The effects of established cytotoxic agents that have an irreversible lethal effect on rapidly dividing cells (1) can be visualized by various methods and are typically monitored by imaging response criteria (e.g., RECIST) after a period of several weeks. For novel anti-neoplastic agents, which are directed to specific targets of the tumour lifecycle (2) and which have a predominantly cytostatic activity, metabolic methods of imaging are increasingly used for early response evaluation in addition.

Crucial aspects of the tumour lifecycle that allow for tumour growth, invasion and metastasis, are, among others, based on angiogenesis and hypoxia $(3,4)$. The induction of new vessels ensures the tumour's supply with nutrients and oxygen as well as the elimination of its metabolic waste products (5). This knowledge has led to the development of therapeutic agents that affect the tumour vasculature, either by having an anti-angiogenic effect [e.g., anti-vascular endothelial growth factor (VEGF) or anti-VEGF-receptor antibodies or small tyrosine kinase inhibitors] $(6,7)$, or by actively destroying tumour vessels, as is the case with vascular disrupting agents (VDA). A third group, the vascular targeting agents (VTA), carries anti-tumour compounds by means of a vasculature-targeting moiety (e.g., antibodies and peptides) into the tumour vasculature (8).

VTA can also carry thrombogenic proteins that activate blood coagulation within tumour vessels with subsequent 
thrombosis and tumour vascular infarction (9). One of these pro-coagulatory proteins is a soluble form of the human tissue factor (TF), also called thromboplastin or factor III. TF is a transmembrane glycoprotein that initiates the coagulation cascade by serving as the cell surface receptor for factor VIIa (10); it is the main initiator of coagulation in vivo (11). TF without its transmembrane domain is called truncated TF (tTF) and only has a marginal thrombogenic effect (12). By fusing the tTF protein, e.g., to antibodies that are directed against various tumour vessel markers and by targeting it to the proximity of tumour vessel endothelium, its original coagulation activity is partly restored $(13,14)$. However, when linking the RGD binding motif for integrins to the $\mathrm{N}$-terminus of the tTF protein, the resultant fusion protein only induces thrombosis in small and medium sized tumour vessels (15).

Molecules, which are primarily expressed on tumour endothelial cells and can hence be used as targets for the peptide sequences RGD (GRGDSP) and NGR (GNGRAHA), encompass several receptors and integrins, e.g., $\alpha v \beta 3$ and aminopeptidase $\mathrm{N}$ (CD13) $(16,17)$. In previous animal experiments it could be shown that tTF proteins, which are C-terminally fused to the RGD or NGR sequence, are capable of initiating tumour vessel thrombosis resulting in an inhibition of tumour growth $(9,12,18)$.

However, since VTA, such as tTF-NGR, have entered early clinical trials in oncology, a detailed understanding of the exact pharmacokinetics and pharmacodynamics of tTF-NGR, as well as an investigation of its early image-based biological effects are very important. In a recent study, the therapeutic effect of tTF-NGR could already be visualized by using single photon emission computed tomography (SPECT), contrast-enhanced ultrasonography (CEUS), fluorescence reflectance imaging (FRI), and ultrasmall superparamagnetic iron oxide magnetic resonance imaging (USPIO-MRI) four to eight hours after treatment initiation $(12,19,20)$. Gadofosveset (MS-325) represents a 'blood pool' MR contrast agent that is approved by the Food and Drug Administration (FDA) and clinically available, and which can be used for MR-angiography (MRA) and dynamic contrast-enhanced MRI (DCE-MRI) (21).

The aim of the animal study reported here was to evaluate the diagnostic potential of DCE-MRI with regard to the early in vivo visualization of the mechanism of action of tTF-NGR. Considering the ongoing clinical application of tTF-NGR, DCE-MRI promises to serve as a simple, quick and well-tolerated imaging biomarker for prompt monitoring of the therapeutic response of malignant tumours to anti-vascular treatment in humans.

\section{Materials and methods}

Cell culture and tumour xenograft model. This study on animals was performed in agreement with government regulations (Deutsches Tierschutzgesetz $§ 8$ Abs. 2) and specifically approved in form of a project license. The protocol was approved by the local committee on the ethics of animal experiments at the LANUV (Landesamt für Natur, Umwelt und Verbraucherschutz) of North Rhine-Westphalia (permit no./project licence: 84-02.04.2012.A247). Athymic CD-1 nude mice for tumour cell transplantation were purchased from
Charles River Laboratories (Sulzfeld, Germany) and acclimated to our animal-experiment facility for at least one week before any experimentation. Mice were maintained in individually ventilated cages (IVC) on a 12:12 h light:dark cycle in a low-stress environment $\left(22^{\circ} \mathrm{C}, 50 \%\right.$ humidity, low noise) and given food and water ad libitum. All MR experiments were performed under isoflurane inhalation $\left(\mathrm{FORENE}^{\circledR}, 2-2.5 \%\right.$; Abbott GmbH \& Co. KG, Wiesbaden, Germany), together with $0.5-1 \mathrm{l} / \mathrm{min} \mathrm{O}_{2}$ ventilation; all efforts were made to minimize suffering.

In this study, we used the human glioblastoma cell line U87 MG [American Type Culture Collection (ATCC); HTB-14; Manassas, VA, USA]. The U87 cell line was cultured in MEM medium supplemented with $10 \%$ fetal calf serum (FCS), pyruvate $(2 \mathrm{mM})$ and non-essential amino acids (2\%). Cells were grown routinely in a monolayer culture at $37^{\circ} \mathrm{C}$ in a $5 \%$ $\mathrm{CO}_{2}$ humidified atmosphere. U87 cells $\left(2 \times 10^{6}\right)$ were inoculated subcutaneously (s.c.) in the anterior right flank of athymic female CD-1 nude mice and allowed to grow until the tumour xenotransplants reached a maximum volume of approximately $800-1,000 \mathrm{~mm}^{3}$.

Recently, it has been reported that the cell line U87 MG is not representing the original glioblastoma cell line established in 1968 at the University of Uppsala. As described by Allen et al (22) and the International Cell Line Authentication Committee (ICLAC), it is a bona fide glioblastoma cell line whose origin is unknown. Nevertheless, this misidentification issue is unlikely to affect the outcomes of our study. The fact that the used cell line, although it is not the original one, clearly has been identified as a cancer/tumour cell line is thus fully consistent with our aim to demonstrate the feasibility to evaluate the initial tumour vessel infarction induced by the tTF-NGR protein by real-time MRI monitoring.

MRI. Image acquisition was performed on a clinical 3.0 Tesla whole body MR system (Achieva; Philips Healthcare, Best, The Netherlands) using a dedicated small animal solenoid coil with a diameter of $40 \mathrm{~mm}$ (Philips Research Europe, Hamburg, Germany), which was positioned upon the MR scanner's patient table. The solenoid coil comprised a heating system in order to keep the body temperature of the mice constant during the MR examination. Although our institution is also in the possession of a 9.4 T MRI scanner for small animals, we decided to conduct the study on a clinical $3.0 \mathrm{~T}$ scanner in order to acquire data that can be easily transferred to a clinical setting, respectively to human beings.

First, a T2-weighted turbo spin echo (TSE) sequence was acquired in coronal sections for the purpose of anatomical orientation (Fig. 1A). Subsequently, a dynamic T1-weighted turbo field echo (TFE) sequence was conducted in transversal sections (TE: $2.3 \mathrm{msec}$; TR: $10 \mathrm{msec}$; NSA: 1; slice thickness: $2 \mathrm{~mm}$; slice gap: $0.4 \mathrm{~mm}$; flip angle: $15^{\circ}$; dynamic phases: 250; dynamic phase time: $7.3 \mathrm{~s}$, resulting in a total scan time of 30:25 min). The T1w TFE sequence included preparation pulses for signal stabilization, which had not been displayed for data analysis.

Tumour treatment. Cloning, expression and purification of the tTF-NGR protein were described in detail earlier $(9,12,16,23)$. The fusion protein tTF-NGR was diluted in phosphate-buffered 

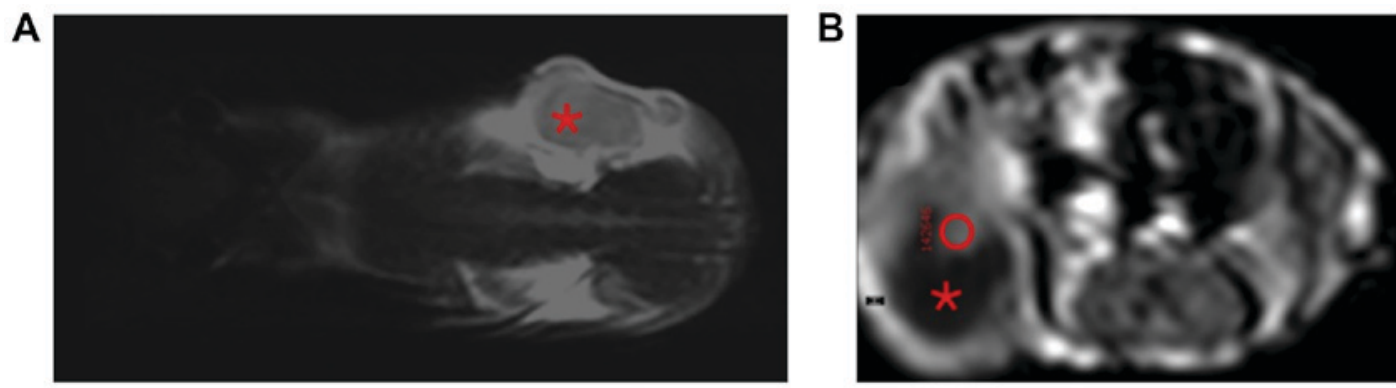

Figure 1. (A) MR sequence for anatomical orientation. T2-weighted, coronally orientated MR image; the tumour in the right flank of the mouse is designated with the asterisk. (B) ROI placement for real-time dynamic gadofosveset-enhanced-MRI analysis. Contrast-enhanced, T1-weighted, axially orientated MR image. The tumour can be seen in the right flank of the mouse (roundish structure in the bottom left-hand corner of the image). A part of the tumour appeared necrotic, i.e., hypointense and non-enhancing in this sequence (asterisk). A ROI has been placed manually into the contrast-enhancing viable part of the tumour (red circle within the hyperintense area). MRI, magnetic resonance imaging; ROI, region of interest.

saline (PBS) and injected intravenously (i.v.) via a tail vein catheter at a dose of $1 \mathrm{mg} / \mathrm{kg}$ body weight (BW) within the MR imaging trials. In the course of the above-mentioned T1-weighted DCE-MRI sequence, $150 \mu \mathrm{l}$ of gadofosveset $(0.25 \mathrm{mmol} / \mathrm{ml})$, a gadolinium-based 'blood pool' contrast agent (MS-325), were injected i.v. via a tail vein catheter. After the first pass and once a 'steady' maximum contrast intensity inside the tumour was obtained, appr. $150 \mu \mathrm{l}$ of tTF-NGR $(1 \mathrm{mg} / \mathrm{kg} \mathrm{BW})$ were injected i.v. via a second tail vein catheter (controls received saline solution $(\mathrm{NaCl})$ instead), and the contrast behaviour of the tumour was observed over the initial period of $30 \mathrm{~min}$.

Within the long-term anti-tumour therapy approach, U87-tumour bearing nude mice were treated systemically by intraperitoneal (i.p.) application of tTF-NGR (1 mg/kg BW; $\mathrm{n}=8$ ) every second day; the control group received $0.9 \%$ saline accordingly $(n=6)$.

Animal study population. A total of 40 tumour-bearing nude mice were included in the MRI study; 16 animals were treated with tTF-NGR and 18 mice served as controls. Six animals did not contribute to the analyses due to technical difficulties, the impossibility of establishing venous access and administering contrast agent and/or tTF-NGR/NaCl, or the animal's death, respectively. During the imaging studies, the animals were anaesthetized by isoflurane inhalation (FORENE ${ }^{\circledR}, 2-2.5 \%$ ), together with $0.5-1 \mathrm{l} / \mathrm{min} \mathrm{O}_{2}$ ventilation.

In order to avoid a bias, which is caused by unique characteristics of the individual mice, four of the animals, which originally served as controls, were subsequently (two days later) used for an additional image acquisition procedure, however then treated with tTF-NGR. This approach enabled the observation of the changes in tumour perfusion following anti-vascular treatment within the same individual as an internal validation of our results.

Analysis of the contrast agent dynamics. Once the acquisition of the MR sequences was completed, the tumour perfusion was analysed by using the software implemented on the MR scanner (IntelliSpace; Philips Healthcare). First of all, the particular slice was chosen, in which the contrast enhancement of the tumour was the most intensive representing an index region of viable tumour tissue with noticeable angiogenesis. In most cases, only a small part of the tumours showed an enhancement, while the major part of the tumours was non-enhancing and necrotic. This is caused by the rapid growth of U87 tumour cells that results in an occasionally inhomogeneous tumour texture. Then, a region of interest (ROI) was placed manually within the contrast-enhancing part of the tumour (exemplarily demonstrated in Fig. 1B). This approach is based on the subjective, individual decision of the investigator, but as mentioned above, it seems to be the best strategy concerning these inhomogeneous tumours in these small animals.

In this way, the individual signal intensity values of this ROI (dimensionless) were acquired over the course of the dynamic MR sequence using IntelliSpace software. Time intensity curves of the acquired 250 dynamic phases (every $7.3 \mathrm{sec}$ ) were automatically plotted and corresponding quantitative values were exported (see also the statistical analysis below). The AUC describes the area under the concentration-time-curve of a pharmaceutical (i.e., contrast agent) in the blood. The area is typically computed starting at the time the contrast agent is administered and ending when the concentration of the pharmaceutical in the blood is negligible. The concentration is measured at different time points and the trapezoidal rule is used to estimate the AUC.

Statistical analysis. Intensity was defined as the brightness of the murine tumours in the course of the dynamic T1-weighted TFE sequence. The signal intensity (SI) values were displayed by the MR system as dimensionless maps and exported in the form of a table. SI was measured in each animal every $7.3 \mathrm{sec}$ (repeated measurements), which defines time points $0(0 \mathrm{sec})$ to 250 , resulting in $1,825 \mathrm{sec}(30 \mathrm{~min}$ and $25 \mathrm{sec}$ ) total duration of the scan. The period from the beginning of the MR sequence (administration of gadofosveset, time point $0 \mathrm{sec}$ ) until the 'steady' maximum intensity of the contrast agent after approximately $496 \mathrm{sec}$ (MR measurement point 68) was disregarded due to the fact that the first pass effect was not of interest in view of the therapeutic response in case of our actually proposed MR technique. Statistical analyses comprised the subsequent dynamic MR-sequence period starting at time point 69 with the injection of tTF-NGR or saline, respectively, and ending at time point $250(=1,825 \mathrm{sec})$ as exemplarily illustrated in Fig. 2A.

To verify the validity of the results and experimental design, two different ways of statistical analyses were 


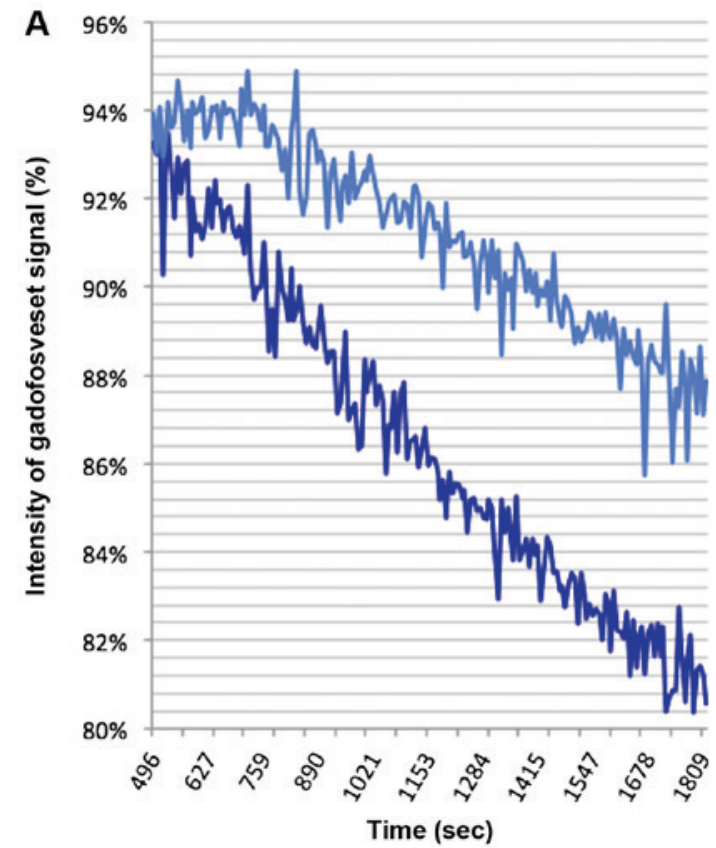

B

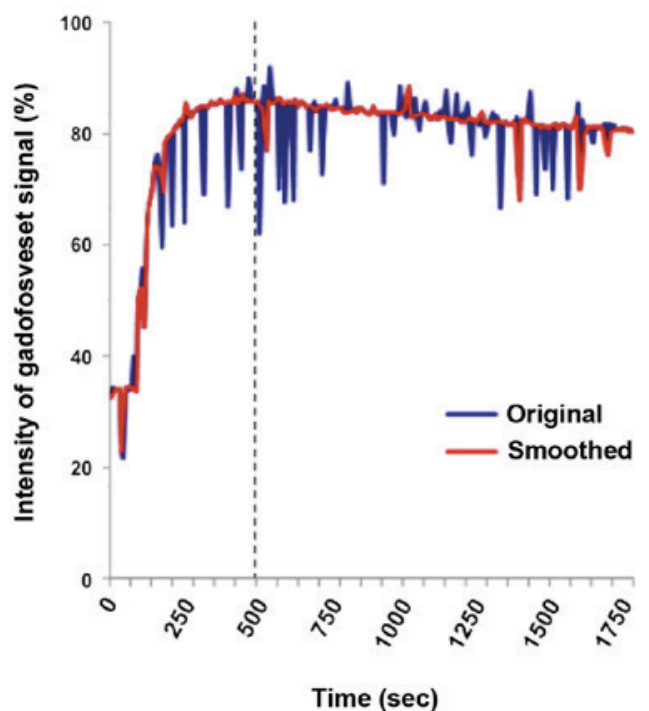

Figure 2. (A) Representative intensity curves of the gadofosveset-enhancing parts of xenotransplants treated with $\mathrm{NaCl}$ (light blue curve), or $1 \mathrm{mg} / \mathrm{kg} \mathrm{BW}$ tTF-NGR (dark blue curve). The intensity curve of the control tumour shows a lesser blood pool-contrast agent wash-out compared to the tTF-NGR-treated tumour, especially demonstrating the stronger decrease of contrast intensity directly after tTF-NGR treatment initiation. (B) Example of an intensity curve prior to and following the smoothing process. Original (blue) and smoothed (red) intensity curves of the contrast-enhancing part of a tumour that was treated with tTF-NGR. Owing to the above-mentioned statistical smoothing method, the breathing artefacts, i.e., spikes of the blue curve, could mostly be eliminated. $\mathrm{tTF}$, truncated tissue factor.

performed. In a first approach, analyses of slope differences of signal intensities like average decrease of intensity between the tTF-NGR and the control group were calculated. This investigation was done by computing the percentage change of the signal intensity of the MR sequence curves at every time point in correlation to the maximum enhancement at time point 69 (injection of tTF-NGR or saline, respectively). In order to analyse the relation between time, group, and intensity trend, a linear mixed model with interaction terms was fitted. An AR1-covariance structure was assumed for the random effects, the corresponding significance level was 0.05 ; any other P-values are considered as explorative. No adjustment for multiple testing was performed, the local significance level is set to 0.05 , P-values $\leq 0.05$ correspond to detected effects. Statistical analyses were performed using SPSS software (IBM corporation, Armonk, NY, USA).

In another approach, the area under the curve (AUC) for the different time periods was computed and compared in detail. For each mouse, the observed SI values were normalized at the time point where tTF-NGR (or $\mathrm{NaCl}$, respectively) was injected. This yields 34 normalized intensity curves, 16 for the tTF-NGR group and 18 for the control group, all having an equal intensity at time point 69 . The AUC from the injection (time point 69) of tTF-NGR or saline, respectively, until the end of the observation period, i.e., the MR sequence, was computed for each animal. Subsequently, the average AUC of the group of mice, which was treated with tTF-NGR, was compared to the average AUC of the control group using a one-sided t-test. The significance level was set to 0.05 , hence $P$-values $\leq 0.05$ correspond to detected effects.

In order to overcome the difficulty that the ROI inside the tumours were exposed to breathing artefacts during the relatively long MR sequence, the intensity curves had to be smoothed (Fig. 2B). Therefore, the relative increases $j_{t}$ in intensity from time point $t$ to time point $t+l$ ('jumps') were computed via $j_{t}=i_{t} / i_{t-1}-1$ (where $i_{t}$ denotes the intensity at time $t$ ). Then, the intensity values were replaced in case two consecutive jumps in opposite direction, larger than a given threshold $\alpha$, were found. This means in exact terms: The intensity values of time $t$ were replaced, if: i) $\left|j_{t}\right|>\alpha$ and $\left|j_{t-1}\right|$ $>\alpha$; ii) $\operatorname{sign}\left(j_{t}\right) \neq \operatorname{sign}\left(j_{t-1}\right)$; and iii) the intensity of $t-1$ was not replaced.

Intensities were replaced by linear interpolation, i.e. replaced intensity of time $t$ was set to $\left(i_{t+1}+i_{t-1}\right) / 2$. Results were computed using different thresholds: $\alpha=1,2$ and 3\%, respectively. The robustness of the obtained results was verified by i) relaxing the assumption of using $500 \mathrm{sec}(8.3 \mathrm{~min})$ as a starting point by instead using 9 and $10 \mathrm{~min}$, respectively (both for normalization and as the AUC starting point); ii) relaxing the assumption of using $30 \mathrm{~min}$ as the end point by instead using 10, 15 and 20 min, respectively; iii) computing the AUC from the time of maximum signal intensity to the end of the observation period (again normalizing at the respective maximum point and furthermore correcting for the time period over which the AUC is computed by an appropriate scaling factor); and iv) relaxing the assumption of equal variances by using a t-test based on unequal variances. Statistical analyses were performed using Excel (Microsoft Corporation, Redmond, WA, USA), the reported results are based on normalized intensities.

Histology. Histological analyses of U87-xenograft tumour tissues were performed with cryo-conserved tissues according to standard protocols. Briefly, tissues were 
Table I. Mixed model analysis, group and time vs. intensity trend. ${ }^{\mathrm{a}}$

\begin{tabular}{llll}
\hline Parameter & Estimate & 95\% confidence interval & P-value \\
\hline Intercept & 0.94651377 & $0.93527749-0.95775005$ & $<0.001$ \\
Intensity decrease in time/sec (control group): $\beta_{\text {time }}$ & -0.00005250 & $-0.06705,-0.03794$ & $<0.001$ \\
Additional group effect (tTF-NGR): $\beta_{\text {tTF }}$ & -0.02049422 & $-0.03687377,-0.00411468$ & 0.014 \\
Additional intensity decrease/sec (tTF-NGR): $\beta_{\text {time tTF }}$ & -0.00004112 & $-0.06233,-0.01991$ & $<0.001$
\end{tabular}

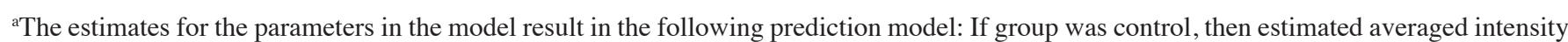
(control group $)=(0.94651377-0.00005250) \times$ time. If group was tTF-NGR, then estimated averaged intensity $($ tTF-NGR group $)=[(0.94651377$ $-0.02049422)-(0.00005250+0.00004112)] \mathrm{x}$ time. $\mathrm{tTF}$, truncated tissue factor.

embedded in Tissue-TEK O.C.T. (Sakura, Alphen aan den Rijn, The Netherlands), snap-frozen in liquid nitrogen, and stored at $-85^{\circ} \mathrm{C}$. Frozen samples were cut to $5 \mu \mathrm{m}$ sections and transferred onto glass slides. For identification of thrombosis, haematoxylin and eosin (H\&E)-stained sections were examined using conventional light microscopy for signs of intratumoral thrombosis, vascular pooling and disruption, and intratumoral haemorrhage.

\section{Results}

Contrast behaviour. After i.v. administration of gadofosveset, all tumours (both in the treated animals and in the controls) showed an intensive contrast enhancement within their perfused, non-necrotic parts; the contrast agent was delivered approximately $70 \mathrm{sec}$ after the beginning of the sequence, and the 'steady' maximum intensity was usually reached after approximately $400 \mathrm{sec}$, at the latest at time point 68-69 (496-500 sec; see Fig. 2A). Saline or tTF-NGR (1 mg/kg bw), respectively, was administered i.v. as soon as the maximum intensity of the contrast agent was reached, approximately $500 \mathrm{sec}$ after the scan was started. The numerous spikes of the measured intensity curves are caused by the respiration movements of the mice's bodies during the relatively long MR sequence. In order to optimize the statistical analyses, these breathing artefacts could mostly be eliminated by the above described smoothing method (Fig. 2B), making the results more robust and ensuring that outliers do not bias the results.

The intensity curves of the gadofosveset signal within the contrast-enhancing parts of the control xenotransplants show a lesser blood pool-contrast agent wash-out compared to the tTF-NGR-treated tumours, as exemplarily demonstrated in Fig. 2A. Especially the stronger decrease of the contrast intensity directly after tTF-NGR treatment initiation in comparison to the controls can be interpreted as a positive therapeutic effect in terms of thrombosis of tumour vessels leading to decreased tumour blood perfusion.

Analysis of slope differences of signal intensity curves. The investigation of the slope difference of signal intensities between the control group and the tTF-NGR group revealed an average decrease of intensity in the control group of -0.00005250 per second $(\mathrm{P}<0.001,95 \% \mathrm{CI}$ : $-0.00006705,-0.00003794$; Table I). In the group of animals that received tTF-NGR, we determined a stronger average decrease of intensity
(-0.00009362 per second). This means that in the tTF-NGR group, the average decrease of intensity is-0.00004112 per second greater than in the control group $(\mathrm{P}<0.001,95 \% \mathrm{CI}$ : $-0.00006705,-0.00003794)$. The differences of the intensities between the control and the tTF-NGR group are displayed as a boxplot illustration in Fig. 3 .

Thus, the contrast intensity within the tumours exhibited a noticeably stronger initial decrease after treating the mice with a therapeutic dose of tTF-NGR compared to the mice that received $\mathrm{NaCl}$ only ( -0.00009362 per second (tTF-NGR) vs. -0.00005250 per second (control)). This corresponds to a $5.67 \%$ additional wash-out effect of the tTF-NGR treated group (12.95\% decrease in contrast intensity between the start and the end of the dynamic MRI course) compared to the control group (only $7.28 \%$ decrease in contrast intensity). This effect is most likely attributable to the therapeutic thrombogenic effect of tTF-NGR within the tumour neovascularization and the consecutive reduction of the tumour perfusion.

Analysis of AUC values of signal intensity curves. Within the more detailed statistical approach, in which the AUC for the different time periods was normalized and smoothed, the intensity curve of the control animals clearly shows a 'steady state' of the blood-pool contrast agent with only a minor decrease, which means that the i.v. application of $\mathrm{NaCl}$ does not have a noticeable effect on the tumours' perfusion (Fig. 4A, red curve). In case of the animals that were treated with tTF-NGR, the intensity curve shows a significantly stronger decrease, i.e., the contrast agent is eliminated from the tumours more quickly (Fig. 4A, blue curve).

Table II displays the results of the t-tests for each of the above-mentioned time periods (see chapter 'statistical analysis') for the threshold $\alpha=1 \%$. The main analysis, being the AUC from the time point where tTF-NGR/NaCl was injected (starting point $8.3 \mathrm{~min}$ ) until the end of the MR sequence (first line in Table II), shows significantly different average AUC values between the two groups at a confidence level of $95 \%$ (Fig. 4B).

Computing the AUC for shorter intervals, i.e., until 20, 15 and 10 min after the injection of the contrast agent, yields similar results, except for the $10 \mathrm{~min}$ case, in which the difference is not significantly different from 0 . This, however, represents a time period of only $1.7 \mathrm{~min}$ (from the time tTF-NGR/ $\mathrm{NaCl}$ was injected). As shown in Table II, the longer the time period considered, the more pronounced the 


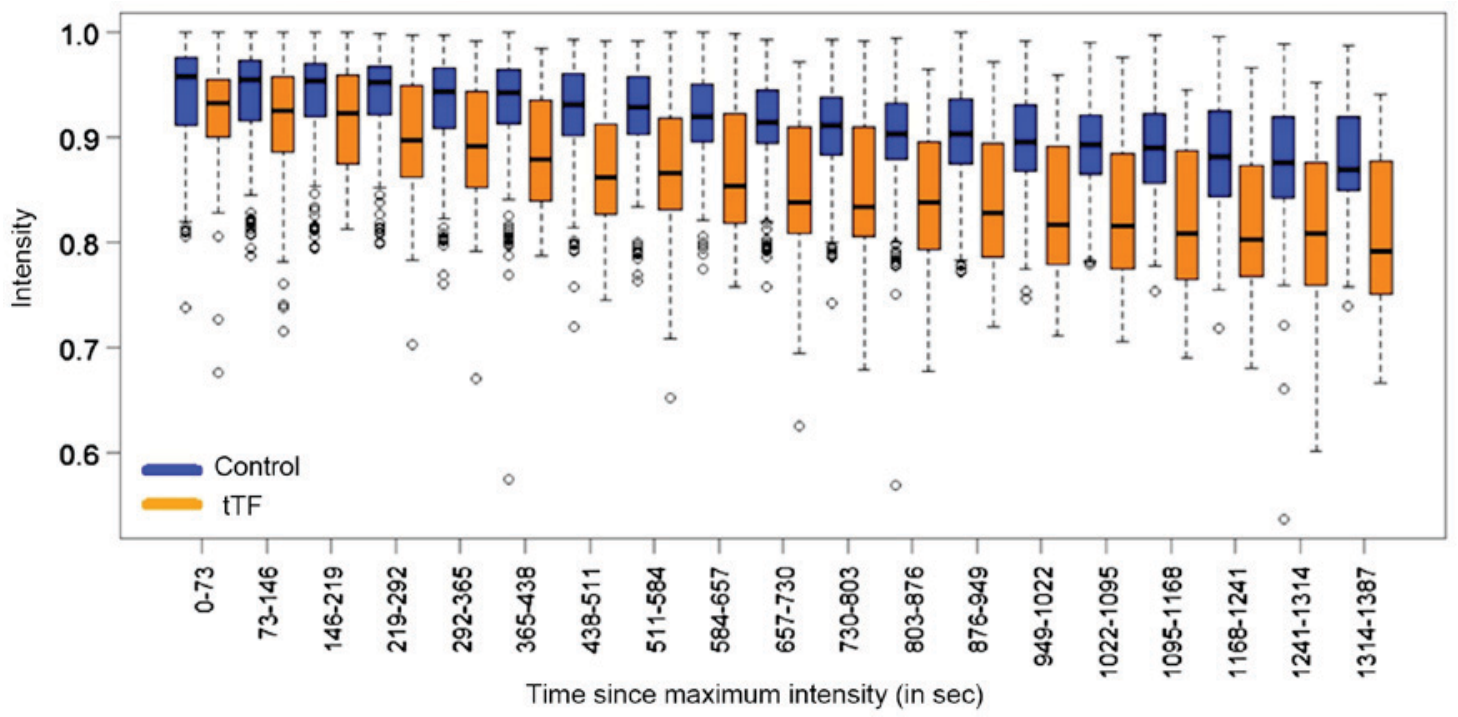

Figure 3. Association between intensity trend and time [control (blue) vs. tTF-NGR (orange)]. Illustration by means of boxplots, outliers displayed as circles. tTF, truncated tissue factor.
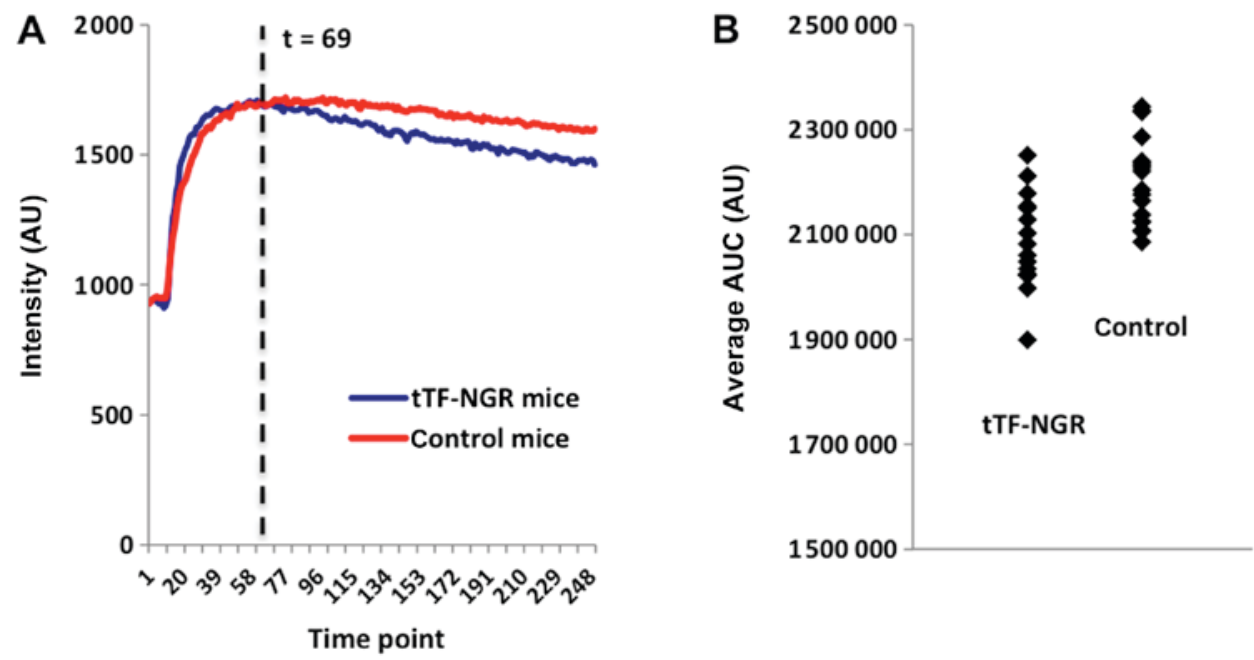

Figure 4. (A) Comparison of the average smoothed intensity curves of the tTF-NGR and the control animals. The intensities were normalized at the time medication was given; the curves represent the smoothed intensities at each time point, averaged across all animals within the control group (red), and averaged across all animals treated with tTF-NGR (blue). Each time point represents a dynamic MR measuring phase (7.3 sec each), which defines time points 0 ( 0 sec) to $250(1,825 \mathrm{sec})$, and time point $69(\sim 500 \mathrm{sec})$. (B) Comparison of the individual AUC values for the time period 8.3 to $30 \mathrm{~min}(\alpha=1 \%)$. Individual AUC values (derived from the smoothed intensity curves) for both the tTF-NGR and the $\mathrm{NaCl}$ group. Please note that for illustration purposes the y-axis does not start at 0 . AUC, area under the curve; tTF, truncated tissue factor.

difference between the respective average AUC values. Using the time point of the maximum signal intensity as a starting point for the calculation of the AUC again yields significantly different AUC averages at a confidence level of $95 \%$.

Table III shows the P-values of the relevant $\mathrm{t}$-tests for both unsmoothed and smoothed curves, based on different thresholds ( $\alpha=1,2$ and $3 \%$, respectively). While in Table II only a smoothing threshold of $\alpha=1 \%$ was applied, Table III shows the respective results for different smoothing thresholds and thus provides evidence of the statistical robustness of the results obtained for $\alpha=1 \%$. In fact, for the main scenario (AUC starting at the time point where tTF-NGR/NaCl was injected) the differences between the AUC of the treated and the control mice are more pronounced using smoothed curves. As shown by Table III, the results are robust with respect to different values of the smoothing threshold $\alpha$ (for $\alpha=2 \%$ the relative difference was $5.2 \%$, for $\alpha=3 \% 5.1 \%$, and for the unsmoothed curves $4.9 \%$, respectively). Taken together, the average AUC values of the intensity curves of the two groups (tTF-NGR vs. control) supply evidence to differ significantly in most cases.

As additional evidence, we used four mice first as controls before they were treated with tTF-NGR in order to eliminate inter-individual differences between the various animals. Except for one animal (mouse no. 86), the AUC was greater in case the mice received $\mathrm{NaCl}$ (Table IV). The results of the smoothed intensity curves of the contrast-enhancing parts of the tumours that were first treated with $\mathrm{NaCl}$ and later with tTF-NGR show a minor decrease, but this decrease is clearly greater following the treatment with tTF-NGR (data not shown). Nevertheless, this approach enabled the observation of the changes in tumour 
Table II. Results of the Student's t-tests in the different AUC. ${ }^{\text {b }}$

\begin{tabular}{|c|c|c|c|c|c|c|c|}
\hline \multicolumn{3}{|c|}{ Scenario } & \multirow{2}{*}{\multicolumn{2}{|c|}{ Average AUC }} & \multirow{2}{*}{\multicolumn{2}{|c|}{ Difference }} & \multirow[b]{3}{*}{ P-value ${ }^{a}$} \\
\hline \multirow{2}{*}{$\begin{array}{l}\text { Normalized, } \\
\text { min }\end{array}$} & \multirow{2}{*}{$\begin{array}{l}\text { AUC from } \\
\text { time, min }\end{array}$} & \multirow{2}{*}{$\begin{array}{l}\text { AUC to } \\
\text { time, min }\end{array}$} & & & & & \\
\hline & & & tTF-NGR & Control & Absolute & Relative (\%) & \\
\hline \multirow[t]{4}{*}{8.3} & 8.3 & 30 & $2,085,311$ & $2,203,500$ & 118,190 & 5.7 & 0.0002 \\
\hline & & 20 & $1,137,358$ & $1,184,841$ & 47,483 & 4.2 & 0.0001 \\
\hline & & 15 & 664,323 & 683,336 & 19,013 & 2.9 & 0.0003 \\
\hline & & 10 & 171,830 & 174,408 & 2,578 & 1.5 & 0.0024 \\
\hline \multirow[t]{4}{*}{9} & 9 & 30 & $2,028,616$ & $2,095,892$ & 67,276 & 3.3 & 0.0398 \\
\hline & & 20 & $1,072,341$ & $1,092,977$ & 20,636 & 1.9 & 0.0997 \\
\hline & & 15 & 595,217 & 599,203 & 3,986 & 0.7 & 0.2969 \\
\hline & & 10 & 98,617 & 98,094 & -522 & -0.5 & 0.2732 \\
\hline \multirow[t]{3}{*}{10} & 10 & 30 & $1,947,814$ & $2,016,251$ & 68,437 & 3.5 & 0.0031 \\
\hline & & 20 & 989,080 & $1,010,287$ & 21,207 & 2.1 & 0.0054 \\
\hline & & 15 & 510,565 & 515,004 & 4,439 & 0.9 & 0.0669 \\
\hline $\begin{array}{l}\text { Max } \\
\text { intensity }\end{array}$ & $\begin{array}{c}\text { Max } \\
\text { intensity }\end{array}$ & 30 & $2,086,301$ & $2,207,055$ & 120,755 & 5.8 & 0.0009 \\
\hline
\end{tabular}

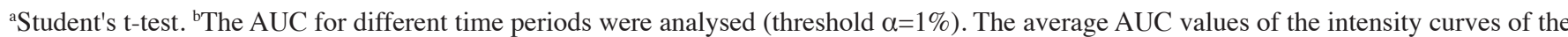
two groups (tTF-NGR vs. control) differ significantly in the majority of cases, except for the cases where only very short time intervals were compared, e.g., the interval between 9 and $10 \mathrm{~min}$. AUC, area under the curve; tTF, truncated tissue factor.

Table III. P-values of the relevant Student's t-tests. ${ }^{\text {b }}$

\begin{tabular}{|c|c|c|c|c|c|c|}
\hline \multicolumn{3}{|c|}{ Scenario } & \multicolumn{4}{|c|}{ P-value } \\
\hline $\begin{array}{l}\text { Normalized, } \\
\min \end{array}$ & $\begin{array}{l}\text { AUC from time, } \\
\text { min }\end{array}$ & $\begin{array}{l}\text { AUC to time, } \\
\text { min }\end{array}$ & Unsmoothed & $\begin{array}{l}\text { Smoothed } \\
\quad \alpha=1 \%\end{array}$ & $\begin{array}{c}\text { Smoothed } \\
\alpha=2 \%\end{array}$ & $\begin{array}{l}\text { Smoothed } \\
\quad \alpha=3 \%\end{array}$ \\
\hline \multirow[t]{4}{*}{8.3} & \multirow[t]{4}{*}{8.3} & 30 & 0.0005 & 0.0002 & 0.0003 & 0.0004 \\
\hline & & 20 & 0.0004 & 0.0001 & 0.0002 & 0.0002 \\
\hline & & 15 & 0.0013 & 0.0003 & 0.0006 & 0.0006 \\
\hline & & 10 & 0.1219 & 0.0024 & 0.0054 & 0.0081 \\
\hline \multirow[t]{4}{*}{9} & \multirow[t]{4}{*}{9} & 30 & 0.0429 & 0.0398 & 0.0359 & 0.0643 \\
\hline & & 20 & 0.0881 & 0.0997 & 0.0853 & 0.1326 \\
\hline & & 15 & 0.1581 & 0.2969 & 0.2327 & 0.2830 \\
\hline & & 10 & 0.3525 & 0.2732 & 0.3688 & 0.3404 \\
\hline \multirow[t]{3}{*}{10} & \multirow[t]{3}{*}{10} & 30 & 0.0019 & 0.0031 & 0.0079 & 0.0052 \\
\hline & & 20 & 0.0023 & 0.0054 & 0.0167 & 0.0082 \\
\hline & & 15 & 0.0229 & 0.0669 & 0.2070 & 0.1114 \\
\hline Max intensity & Max intensity & 30 & 0.0050 & 0.0009 & 0.0032 & 0.0030 \\
\hline
\end{tabular}

${ }^{\text {aS }}$ Student's t-test. ${ }^{\mathrm{b} P}$-values of the relevant t-tests for unsmoothed (i.e., original) and for smoothed curves, based on the thresholds $\alpha=1,2$ and $3 \%$, respectively.

perfusion following anti-vascular treatment within the same individual as an internal validation of our results and thus the mode of action of the tTF-NGR therapy.

Anti-tumour effects of the tTF-NGR therapy. The aim of this study was to prove for the potential of the gadofosveset-based real-time DCE-MRI in imaging the initial therapeutic effect of the anti-vascular tTF-NGR approach, which has been described in various tumour models $(9,12,16,19,23)$. Because of this, no direct correlation of the in vivo imaging data with tumour histology has been performed. However, representative photographs of tTF-NGR-treated and gadofosveset-imaged U87-tumour slides show extensive blood pooling with subsequent vascular disruption due to thrombosis of blood vessels 
Table IV. Intra-individual differences in the AUC. ${ }^{a}$

\begin{tabular}{lccrrr}
\hline & \multicolumn{2}{c}{ AUC } & & \multicolumn{2}{c}{ Difference } \\
\cline { 2 - 3 } \cline { 5 - 6 } Mouse & tTF-NGR & Control & & Absolute & Relative (\%) \\
\hline no. 86 & $2,858,121$ & $2,854,763$ & & -3358 & -0.1 \\
no. 89 & $2,716,574$ & $2,877,707$ & & 161,133 & 5.9 \\
no. 90 & $2,805,003$ & $2,963,184$ & & 158,181 & 5.6 \\
no. 2219 & $2,751,362$ & $2,822,809$ & & 71,447 & 2.6 \\
\hline
\end{tabular}

antra-individual differences in the AUC values in four exemplary mice that received $\mathrm{NaCl}$ first and were subsequently treated with tTF-NGR. AUC, area under the curve; tTF, truncated tissue factor.

(Fig. 5) while control tumours exhibit no thrombosis (data not shown).

In order to also test for the long-term therapeutic effect of tTF-NGR on U87 glioblastoma, xenotransplant-bearing CD-1 nude mice were systematically treated with tTF-NGR every second day for two weeks. Tumour growth was significantly reduced by i.p. application of tTF-NGR compared to the saline control group (Fig. 6).

\section{Discussion}

tTF-NGR is an innovative VTA that has been shown to be effective in experimental cancer treatment, as it induces thrombosis in tumour vessels and thereby leads to an inhibition of tumour growth $(9,12,16,18,19,23-25$; Figs. 5 and 6). The therapeutic effect of tTF-NGR has already been visualized by different radiological and nuclear medicine procedures four to eight hours after VTA therapy initiation (18), but the clinically applicable in vivo-visualization of its initial effectiveness in real-time by gadofosveset-enhanced MRI has been pending so far. The real-time visualization and early assessment of the therapeutic effectiveness of tTF-NGR by means of an imaging procedure that can be easily transferred to humans is particularly essential with regard to its clinical implementation, since an objective and fast imaging biomarker for monitoring the thrombogenic effects of tTF-NGR, i.e., the therapeutic response of malignant diseases, is indispensable for an adequate cancer treatment regimen (26). Furthermore, potential non-responders could be timely identified.

The histopathological, i.e., ex-vivo-measurement of vessel density is a reference standard in order to determine the vascularization rate of a tumour. But since this method has the disadvantage of invasiveness, dynamic contrast-enhanced MRI (DCE-MRI) is the imaging modality most frequently used in preclinical and clinical studies to assess the effect of VTA and VDA $(27,28)$. In this manner, the anti-vascular effect can be monitored non-invasively by using different kinetic parameters (e.g., $\mathrm{K}^{\text {trans }}$ ) (29). However, both a robust measurement of the vascular input function (VIF) and the T1-mapping for adequate pharmacokinetic modelling are not standardized yet and are beyond that extremely challenging in mice.

In the present animal study, we investigated the mere contrast intensity within the tumours using a 'blood pool' contrast agent in a dynamic T1-weighted TFE-sequence, a potentially simple and robust parameter in DCE-MRI, due to the fact that kinetic parameters for the analysis of the vessel permeability (such as $\mathrm{K}^{\text {trans }}$ ) could not be used for 'blood pool' contrast agents, which represent an intravascular distribution without relevant vessel leakage. The underlying assumption is that viable tumour parts demonstrate an intense 'steady' contrast enhancement following the i.v. administration of a 'blood pool' contrast agent (gadofosveset), while effective tTF-NGR treatment results in a clear reduction of the tumour's perfusion and therefore its contrast enhancement.

Gadofosveset (MS-325) represents an FDA approved 'blood pool' contrast agent that was previously marketed as Vasovist ${ }^{\circledR}$ (Bayer Schering Pharma AG, Berlin, Germany), but which is presently not available anymore. Likewise, gadofosveset trisodium (ABLAVAR ${ }^{\circledR}$; Lantheus Medical Imaging, Inc., North Billerica, MA, USA) was recently withdrawn by its manufacturer due to poor sales, besides it is no longer authorized in the EU. Nevertheless, other i.v. contrast agents such as gadobenate dimeglumine (MultiHance ${ }^{\circledR}$; Bracco Imaging $\mathrm{GmbH}$, Konstanz, Germany), which has a comparatively high protein binding and therefore shows characteristics of 'blood pool' contrast agents, may possibly be used as contrast agent for real-time MRI of the tumour response to anti-vascular drugs. This possibility should be subject to future research, even though in the EU, MultiHance ${ }^{\circledR}$ is currently only approved for MR imaging of the liver (30). ABLAVAR $^{\circledR}$ and Vasovist ${ }^{\circledR}$ are 'blood pool' contrast agents demonstrating a contrast behaviour that is analogous to the behaviour of long circulating USPIO. The potential of USPIO in terms of the visualization of the therapeutic effect of anti-vascular substances has already been shown (31), but USPIO are presently not clinically approved for imaging in humans.

The aim of this study was to examine the potential of gadofosveset-based real-time DCE-MRI in evaluating the initial mechanism of action (MOA) of anti-vascular tTF-NGR. By investigation of the slope difference of signal intensities, we could show that the contrast intensity within the tumours, measured only for approximately 23 min following the application of tTF-NGR or control vehicle, respectively, reflecting the initial short-time effect of tTF-NGR, exhibited a statistically significantly stronger decrease when treating the mice with a therapeutic dose of tTF-NGR compared to the mice that received $\mathrm{NaCl}$ only. This corresponds to a $5.67 \%$ additional wash-out effect of the tTF-NGR-treated group: $12.95 \%$ decrease in contrast intensity between the start and the end of the 1,387-sec dynamic MRI course compared to the control group with only $7.28 \%$ decrease in contrast intensity. The main analysis of the differences between the AUC of the treated and the control mice from the time point where tTF-NGR/NaCl was injected (starting point $8.3 \mathrm{~min}$ ) until the end of the MR sequence (see first line in Table II) shows significantly different average AUC values between the two groups at a confidence level of $95 \%$, and the results are more pronounced using smoothed curves (Fig. 4B).

In summary, both statistical evaluations prove for the thrombogenic effect of tTF-NGR within the tumour neovascularization directly leading to a haemorrhagic infarction of the tumour tissue (Fig. 5) and simultaneously a reduced 


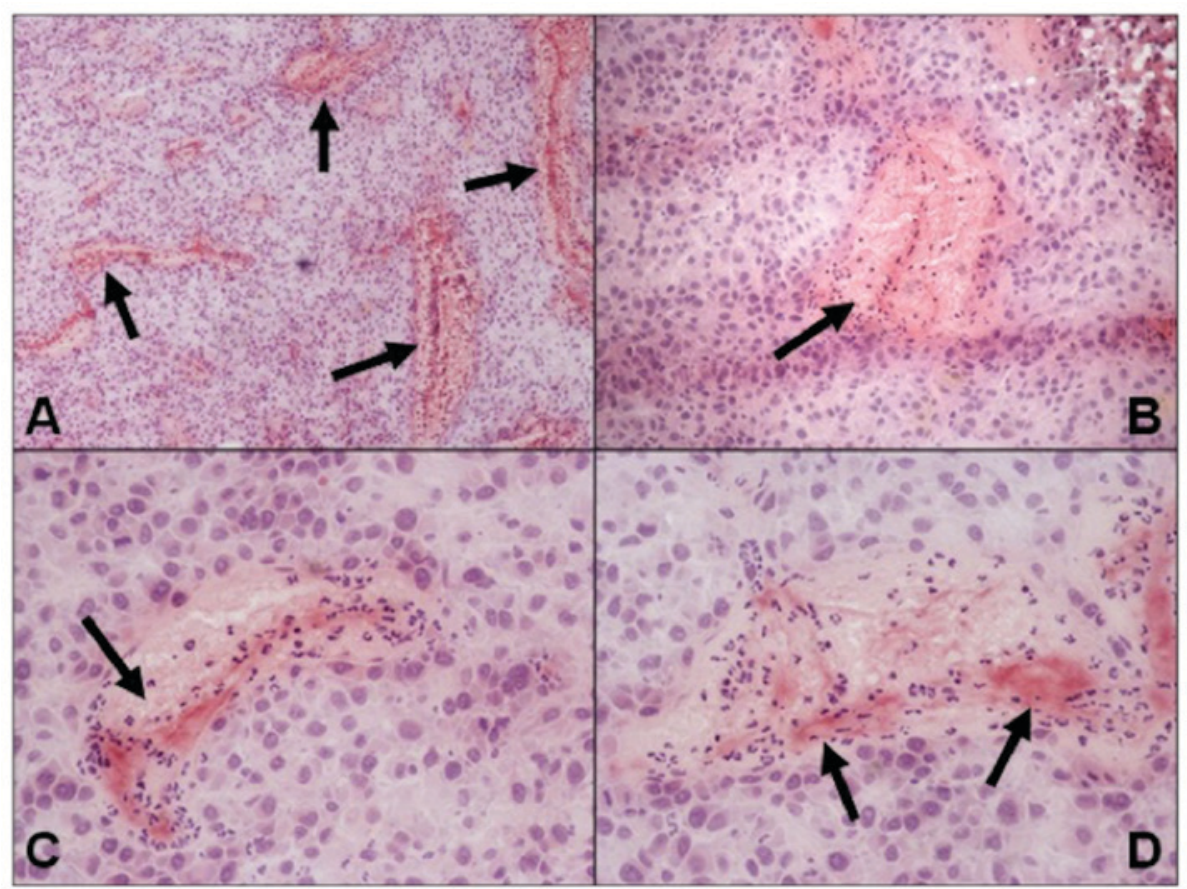

Figure 5. Representative photographs of tTF-NGR-treated and gadofosveset-imaged U87-tumour xenotransplants. Haematoxylin and eosin staining revealed extensive blood pooling with subsequent vascular disruption due to thrombosis of blood vessels (arrows). Magnifications, (A) x40, (B) x100 and (C and D) x200. $\mathrm{tTF}$, truncated tissue factor.

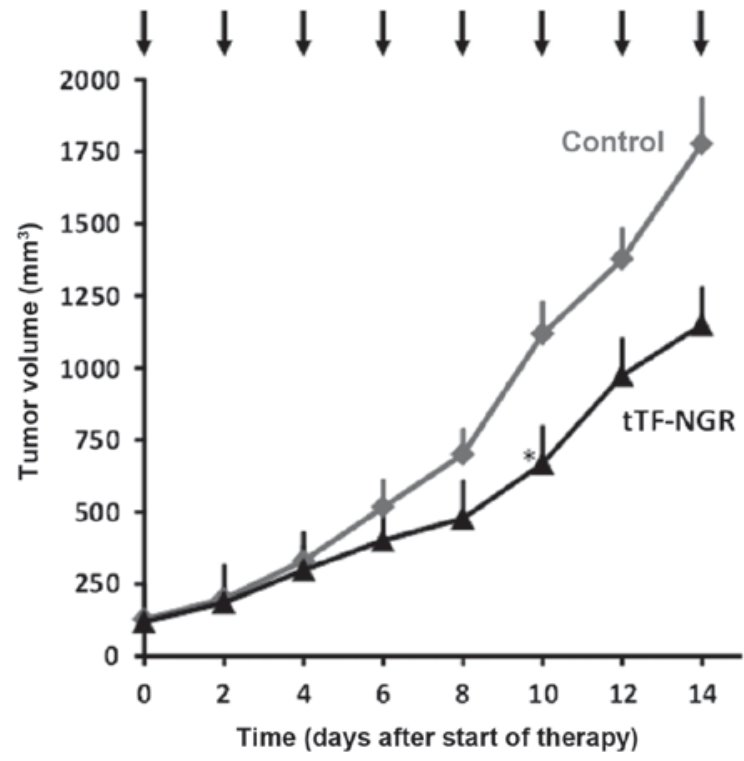

Figure 6. In vivo-therapeutic activity of tTF-NGR against human U87-glioblastoma xenografts. Upon repeated systemic application of tTF-NGR in CD-1 nude mice ( $\mathrm{n}=8 ; 1 \mathrm{mg}$ tTF-NGR/kg body weight $\mathrm{x} 8$; intraperitoneal injection every second day, see arrows), tumour growth of subcutaneous U87 xenotransplants was significantly reduced compared with the saline control group $(n=6)$. Data are presented as the means; the asterisk denotes the first day of significance ( $t$-test, $\mathrm{P} \leq 0.05$ ). tTF, truncated tissue factor.

tumour perfusion. As we measured only for approximately 23 min following the application of tTF-NGR or control vehicle, respectively, our measurements only reflect the initial short-time effect of tTF-NGR, which as the curves separated, became bigger over time. Our finding concerning the MOA of tTF-NGR is also substantiated by the fact that we were able to show these differences in the behaviour of the contrast intensity within one and the same animals following sham and tTF-NGR therapy, respectively, which eliminates possibly existing inter-individual differences between various animals. The fact that the mice in the control group also showed a minor decrease in contrast intensity is related to the physiological renal elimination of the contrast agent. Both the quantification of the amount of vascular infarction induced by tTF-NGR and the characterization of its maximum are beyond the scope of this MR evaluation study, since we could only observe the effect over a short initial time course. In MR imaging performed earlier with USPIO and 4-8 $\mathrm{h}$ after application of tTF-NGR, we have observed a considerably larger decrease of the vascular volume fraction inside the tumours treated with tTF-NGR in comparison to saline controls $(12,19)$.

Further desirable investigations beyond the scope of this study are the comparison of DCE-MRI with additional MR sequences such as diffusion-weighted imaging (DWI), as this method allows for an early quantification of the changes within the tumour tissue (cellularity, oedema and microperfusion) following VTA treatment $(31,32)$, the absolute quantification of the T1/R1 relaxation by modern $\mathrm{T} 1$ mapping, and the correlation of our MRI results with the actual histopathological changes within the tumours following tTF-NGR treatment. In addition, other sequences, e.g., giving tTF-NGR for vessel occlusion before gadofosveset and subsequently measuring block of contrast tumour uptake might be interesting to study for clinical application. Indeed, we have started a phase I trial with tTF-NGR applied as an one-hour infusion via a central venous line in late stage cancer patients and we plan to perform randomised phase II trials with the combination chemotherapy +/- tTF-NGR (24). These trials will be guided by repeated MR examinations to assess for blood flow 
decrease in tumour signal lesions envisaging us to transfer the gadofosveset-enhanced DCE-MRI as real-time assessment of the therapeutic effectiveness of tTF-NGR in order to monitor its thrombogenic effects, to identify potential non-responders etc. at an early stage of the tTF-NGR therapy.

In summary, we have shown that dynamic gadofosveset-enhanced MRI enables an early in vivo visualization of the MOA of anti-vascular drugs in malignant tumours in real-time. In respect of the clinical use of tTF-NGR, gadofosveset-enhanced DCE-MRI should be further studied as a simple, quick and non-invasive imaging technique for monitoring the therapeutic response and an easy-to-handle surrogate biomarker for the treatment surveillance in individuals.

\section{Acknowledgements}

Not applicable.

\section{Funding}

The present study was supported by grants from the Deutsche Krebshilfe (grant no. DKH 110886), the Else-Kröner-Fresenius-Stiftung (grant no. 2013_A284), the Deutsche Forschungsgemeinschaft (grant no. Cluster of Excellence EXC 1003-Cells in Motion), the Sybille-Hahne-Stiftung, and the Innovative Medizinische Forschung (IMF, Münster).

\section{Availability of data and materials}

The datasets used and/or analysed during the current study are available from the corresponding author on reasonable request.

\section{Authors' contributions}

AH performed the MRI scans, collected and interpreted the data and wrote the manuscript. TP, RM, WB and CB designed the study. RK and MB performed the statistical analyses. WH critically reviewed the manuscript for important intellectual content, made substantial contributions to the study's conception and enabled the realisation of the MR experiments and the collaboration between the Departments of Radiology and Oncology. CS designed the study, was responsible for the care and treatment of the animals, and collected and interpreted the data. All authors read and approved the final manuscript.

\section{Ethics approval and consent to participate}

The present study on animals was performed in agreement with German regulations (Tierschutzgesetz $\$ 8 A b s .2$ ) and specifically approved in the form of a project license. The protocol was approved by the local committee on the Ethics of Animal Experiments at the LANUV (Landesamt für Natur, Umwelt und Verbraucherschutz) of North Rhine-Westphalia (permit no./project license: 84-02.04.2012.A247).

\section{Patient consent for publication}

Not applicable.

\section{Competing interests}

The authors declare that they have no competing interests.

\section{References}

1. Gwyther SJ and Schwartz LH: How to assess anti-tumour efficacy by imaging techniques. Eur J Cancer 44: 39-45, 2008.

2. Contractor KB and Aboagye EO: Monitoring predominantly cytostatic treatment response with 18F-FDG PET. J Nucl Med 50 (Suppl 1): 97S-105S, 2009.

3. de Bazelaire C, Alsop DC, George D, Pedrosa I, Wang Y, Michaelson MD and Rofsky NM: Magnetic resonance imaging-measured blood flow change after antiangiogenic therapy with PTK787/ZK 222584 correlates with clinical outcome in metastatic renal cell carcinoma. Clin Cancer Res 14: 5548-5554, 2008.

4. Padhani AR, Krohn KA, Lewis JS and Alber M: Imaging oxygenation of human tumours. Eur Radiol 17: 861-872, 2007.

5. Carmetliet P: Mechanisms of angiogenesis and arteriogenesis. Nat Med 6: 389-395, 2000.

6. Kerbel RS: Antiangiogenic therapy: A universal chemosensitization strategy for cancer? Science 312: 1171-1175, 2006.

7. Murphy DA, Makonnen S, Lassoued W, Feldman MD, Carter C and Lee WM: Inhibition of tumor endothelial ERK activation, angiogenesis, and tumor growth by sorafenib (BAY43-9006). Am J Pathol 169: 1875-1885, 2006.

8. Thorpe PE: Vascular targeting agents as cancer therapeutics. Clin Cancer Res 10: 415-427, 2004.

9. Kessler T, Schwöppe C, Liersch R, Schliemann C, Hintelmann H, Bieker R, Berdel WE and Mesters RM: Generation of fusion proteins for selective occlusion of tumor vessels. Curr Drug Discov Technol 5: 1-8, 2008.

10. Samad F and Ruf W: Inflammation, obesity, and thrombosis. Blood 122: 3415-3422, 2013.

11. Eisenreich A: Regulation of vascular function on posttranscriptional level. Thrombosis 2013: 948765, 2013.

12. Bieker R, Kessler T, Schwöppe C, Padró T, Persigehl T, Bremer C, Dreischalück J, Kolkmeyer A, Heindel W, Mesters RM and Berdel WE: Infarction of tumor vessels by NGR-peptide directed targeting of tissue factor. Experimental results and first-in-man experience. Blood 113: 5019-5027, 2009.

13. Nilsson F, Kosmehl H, Zardi L and Neri D: Targeted delivery of tissue factor to the ED-B domain of fibronectin, a marker of angiogenesis, mediates the infarction of solid tumors in mice. Cancer Res 61: 711-716, 2001.

14. Liu C, Huang H and Doñate F: Prostate-specific membrane antigen directed selective thrombotic infarction of tumors. Cancer Res 62: 5470-5475, 2002.

15. Hu P, Yan J, Sharifi J, Bai T, Khawli LA and Epstein AL: Comparison of three different targeted tissue factor fusion proteins for inducing tumor vessel thrombosis. Cancer Res 63: 5046-5053, 2003.

16. Schwöppe C, Kessler T, Persigehl T, Liersch R, Hintelmann H, Dreischalück J, Ring J, Bremer C, Heindel W, Mesters RM and Berdel WE: Tissue-factor proteins induce occlusion of tumor vessels. Thromb Res 125 (Suppl 2): S143-S150, 2010.

17. Pasqualini R, Koivunen E, Kain R, Lahdenranta J, Sakamoto M, Stryhn A, Ashmun RA, Shapiro LH, Arap W and Ruoslahti E: Aminopeptidase $\mathrm{N}$ is a receptor for tumor-homing peptides and a target for inhibiting angiogenesis. Cancer Res 60: 722-727, 2000.

18. Kessler T, Bieker R, Padró T, Schwöppe C, Persigehl T, Bremer C, Kreuter M, Berdel WE and Mesters RM: Inhibition of tumor growth by RGD peptide-directed delivery of truncated tissue factor to the tumor vasculature. Clin Cancer Res 11: 6317-6324, 2005.

19. Persigehl T, Ring J, Bremer C, Heindel W, Holtmeier R, Stypmann J, Claesener M, Hermann S, Schäfers M,Zerbst C, et al: Non-invasive monitoring of tumor-vessel infarction by retargeted truncated tissue factor tTF-NGR using multi-modal imaging. Angiogenesis 17: 235-246, 2014.

20. Persigehl T, Matuszewski L, Kessler T, Wall A, Meier N, Ebert W, Berdel WE, Heindel W, Mesters R and Bremer C: Prediction of antiangiogenic treatment efficacy by iron oxide enhanced parametric magnetic resonance imaging. Invest Radiol 42: 791-796, 2007. 
21. Sabach AS, Bruno M, Kim D, Mulholland T, Lee L, Kaura S and Lim RP: Gadofosveset trisodium: Abdominal and peripheral vascular applications. AJR Am J Roentgenol 200: 1378-1386, 2013.

22. Allen M, Bjerke M, Edlund H, Nelander S and Westermark B: Origin of the U87MG glioma cell line: Good news and bad news. Sci Transl Med 8: 354re3, 2016.

23. Schwöppe C, Zerbst C, Fröhlich M, Schliemann C, Kessler T, Liersch R, Overkamp L, Holtmeier R, Stypmann J, Dreiling A, et al: Anticancer therapy by tumor vessel infarction with polyethylene glycol conjugated retargeted tissue factor. J Med Chem 56: 2337-2347, 2013.

24. Persigehl T, Bieker R, Matuszewski L, Wall A, Kessler T, Kooijman H, Meier N, Ebert W, Berdel WE, Heindel W, et al: Antiangiogenic tumor treatment: Early noninvasive monitoring with USPIO-enhanced MR imaging in mice. Radiology 244: 449-456, 2007.

25. Stucke-Ring J, Ronnacker J, Brand C, Höltke C, Schliemann C, Kessler T, Schmidt LH, Harrach S, Mantke V, Hintelmann H, et al: Combinatorial effects of doxorubicin and retargeted tissue factor tTF-NGR by intratumoral entrapment of doxorubicin and proapoptotic increase of tumor vascular infarction. Oncotarget 7: 82458-82472, 2016.

26. Höink AJ, Heindel W and Buerke B: Radiological evaluation of the therapeutic response of malignant diseases: Status quo, innovative developments and requirements for radiology. Rofo 186 927-936, 2014
27. Zweifel M and Padhani AR: Perfusion MRI in the early clinical development of antivascular drugs: Decorations or decision making tools? Eur J Nucl Med Mol Imaging 37 (Suppl 1): S164-S182, 2010.

28. Salmon BA, Salmon HW and Siemann DW: Monitoring the treatment efficacy of the vascular disrupting agent CA4P. Eur J Cancer 43: 1622-1629, 2007.

29. Kim KW, Lee JM, Jeon YS, Lee IJ, Choi Y, Park J, Kiefer B, Kim C, Han JK and Choi BI: Vascular disrupting effect of CKD-516: Preclinical study using DCE-MRI. Invest New Drugs 31: 1097-1106, 2013.

30. European Medicines Agency: EMA's final opinion confirms restrictions on use of linear gadolinium agents in body scans. http://www.ema.europa.eu/ema/index.jsp?curl=pages/ news_and_events/news/2017/07/news_detail_002780.jsp\&mid= WC0 $0101 \mathrm{ac} 058004 \mathrm{~d} 5 \mathrm{c} 1$. Accessed Jul $21,201 \overline{7}$.

31. Rezai P, Pisaneschi MJ, Feng C and Yaghmai V: A radiologist's guide to treatment response criteria in oncologic imaging: Functional, molecular, and disease-specific imaging biomarkers. AJR Am J Roentgenol 201: 246-256, 2013.

32. Thoeny HC and Ross BD: Predicting and monitoring cancer treatment response with diffusion-weighted MRI. J Magn Reson Imaging 32: 2-16, 2010.

(i) (9) This work is licensed under a Creative Commons

Cy NC ND Attribution-NonCommercial-NoDerivatives 4.0 International (CC BY-NC-ND 4.0) License. 